\title{
Heavy Flavours in Deep Inelastic Scattering
}

\author{
Marc-Oliver Boenig ${ }^{1}$ \\ on behalf of the $\mathrm{H} 1$ and ZEUS Collaborations \\ 1 - Universität Dortmund, Experimentelle Physik V, \\ Otto-Hahn Str. 4, 44221 Dortmund, Germany
}

\begin{abstract}
In the talk [1] an overview of recent measurements of charm and beauty production in deep inelastic ep collisions at HERA performed by the $\mathrm{H} 1$ and ZEUS experiments has been presented. Various techniques how to identify charm and beauty production are explained, which are based on different final states. The results of the measurements are compared with different QCD calculations for charm and beauty production. Double differential measurements of charm and beauty production are used to extract heavy flavour contributions $F_{2}^{c \bar{c}}$ and $F_{2}^{b \bar{b}}$ to the proton structure function $F_{2}$.
\end{abstract}

\section{Introduction}

Measurements of heavy quark production in deep inelastic electron proton scattering at the HERA collider are well suited to study quantumchromodynamics (QCD). The heavy charm and beauty quarks are produced predominantly by the boson gluon fusion process (BGF). Therefore the measurement of heavy quarks is directly sensitive to the gluon density of the proton. The high mass of the quarks, $m_{c}$ and $m_{b}$, allows predictions by the means of pertubative QCD (pQCD). Such calculations can be performed in the massive scheme $^{\mathrm{a}}$ (e.g. HVQDIS), which are expected to be valid at $m_{c / b} \sim Q^{2}$. In contrast the massless calculation is expected to be valid at $m_{c / b} \ll Q^{2}$. The intermediate scheme ${ }^{b}$ uses at low $Q^{2}$ the massive and at high $Q^{2}$

\footnotetext{
${ }^{a}$ Fixed flavour numbering sheme (FFNS)

bVariable flavour numbering scheme (VFNS)
}

the massless approach and interpolates in the transition region.

Deep inelastic scattering (DIS) is defined as the four momentum transfer squared $Q^{2}>$ $2 \mathrm{GeV}^{2}$. $\mathrm{H} 1$ and ZEUS select these kind of events by reconstructing the scattered electron in the calorimeters which surround the inner tracking detectors.

\section{Charm Production}

Events with the production of charm quarks can be identified by reconstruction of resonance decays e.g. of the charged $D^{\star}$ meson via: $D^{\star \pm} \rightarrow D^{0} \pi_{\text {slow }}^{ \pm} \rightarrow K^{\mp} \pi^{ \pm} \pm_{\text {slow }}$. Using in addition the lifetime information from silicon vertex detectors it is possible to improve the signal of other $D$ meson decays like: $D^{ \pm} \rightarrow K \pi^{+} \pi^{-}+$c.c. in order to perform a measurement. This measurement has been performed by the ZEUS collaboration in the visible phase space of $5<Q^{2}<1000 \mathrm{GeV}^{2}$ in $Q^{2}$, in the inelasticity $y$ of scattering process from 0.02 to 0.7 , in the pseudorapidity $\left|\eta\left(D^{ \pm}\right)\right|<1.6$ and in the transverse momentum $3<p_{t}\left(D^{ \pm}\right)<20 \mathrm{GeV}$ as shown in Figure 1 as a function of $p_{t}\left(D^{ \pm}\right)$[2]. The measurement is compared to the massive NLO calculation, which is able to describe the data well.

The $D^{\star}$ measurement by the $\mathrm{H} 1$ collaboration [3] is shown in the Figure 2 as a function of the pseudorapidity $\eta\left(D^{\star}\right)$ in the visible phase space of $5-100 \mathrm{GeV}^{2}, 0.05<y<0.6$, $\left|\eta\left(D^{\star}\right)\right|<1.5$ and $p_{t}\left(D^{\star}\right)>1.5 \mathrm{GeV}$ is reasonably well described by the Monte Carlo programs RAPGAP and CASCADE. The HVQDIS prediction reveals a discrepancy in the forward direction. A double differential 


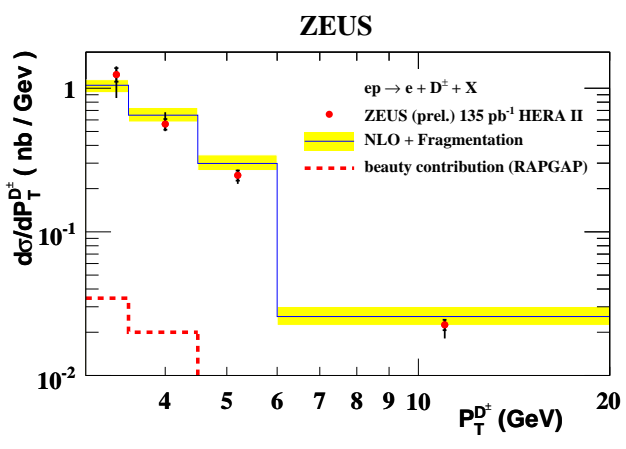

Figure 1: Differential cross section for the $D^{ \pm}$production as a function of $p_{t}\left(D^{ \pm}\right)[2]$.

measurement shows that this discrepancy is localised at low $p_{t}\left(D^{\star}\right)$.

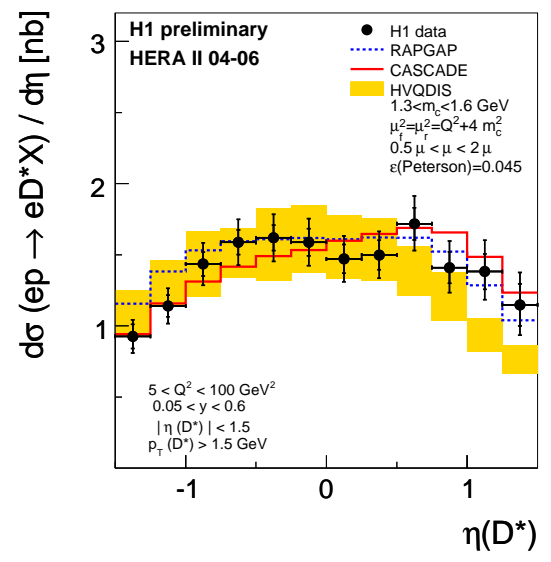

Figure 2: Differential cross section for the $D^{\star}$ production as a function of $\eta\left(D^{\star}\right)[3]$.

This discrepancy between the data and the NLO prediction is not seen in the $D^{ \pm}$measurement by ZEUS [2], where the structure functions ZEUS NLO PDF has been used in contrast to CTEQ5F3, which has been used by $\mathrm{H} 1$ for the NLO prediction. On the other side the use of the ZEUS NLO PDF leads to a worse description of the data as a function of Bjorken $x$ compared to the H1 measurement.

While the hard scattering process for heavy flavour production is calculable with the means of $\mathrm{pQCD}$, the fragmentation of the quarks to hadrons is modelled using phenomenological models. One of these models is the Peterson fragmentation, which has the free parameter $\epsilon_{Q}$ to be determined by experiment. The fragmentation function is used in a model with leading order matrix elements and matched parton showers, string fragmentation and particles decays as implemented in the MC program PYTHIA. The measurement by $\mathrm{H} 1$ using the hemisphere method and reaching down close to the production threshold of the charm quarks gives $\epsilon_{Q}=0.018 \pm_{0.003}^{0.004}$ [4]. The jet method selects events with higher energies, due to the jet requirement, and yields $\epsilon_{Q}=0.030 \pm_{0.005}^{0.006}$ [4]. The ZEUS measurement has a harder jet requirement and results in $\epsilon_{Q}=0.064 \pm$ $0.006_{-0.008}^{+0.011}[5]$. The different results obtained are likely to be caused different kinematical region selected by the different methods.

\section{Beauty Production}

One method to identify beauty production in DIS is to select events with at least one jet and one muon associated with one of the jets. If the muon originates from a decay of a B meson the muon has on average a higher relative transverse momentum $p_{t}^{r e l}$ to the corresponding jet in comparison to muons originating from light quarks. The amount of beauty production is determined using template distributions for $b$ and light quarks from Monte Carlo predictions and fitting their normalisation such that the $p_{t}^{r e l}$ distribution in data is described best by the normalised sum. The result of such a measurement performed by the ZEUS collaboration [6] is shown in Figure 3 as a function of the photon virtuality $Q^{2}$ in the visible phase space of $Q^{2}>4 \mathrm{GeV}^{2}$, $0.05<y<0.6, E_{\mathrm{t}, \text { jet }}>5 \mathrm{GeV},-2<\eta_{\text {jet }}<$ $2.5, p_{t, \mu}>1.5 \mathrm{GeV}$ and $-1.6>\eta_{\mu}$. The result is compared to the prediction by RAPGAP (scaled by 2.49) and a massive NLO prediction; both prediction can describe the shape but not the normalisation. 


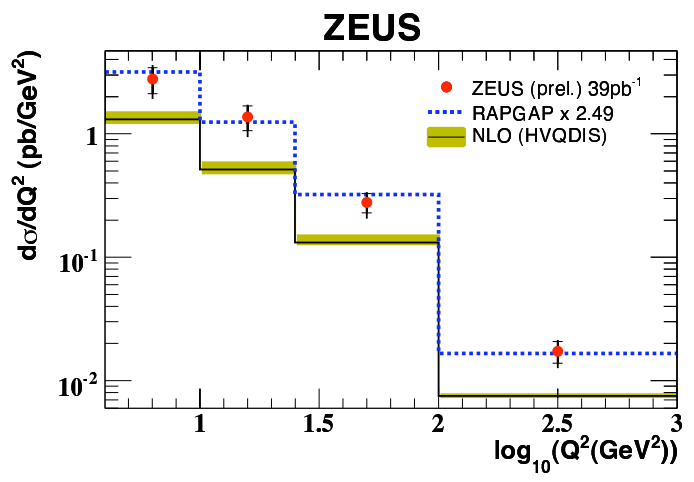

Figure 3: Differential cross section for beauty production as a function of the photon virtuality $Q^{2}[6]$.

Using this method double differential cross section in $x$ and $Q^{2}$ have been measured and used to extract the proton structure function $F_{2}^{b \bar{b}}$ after extrapolation the measurement to full kinematic phase space. The inclusive cross section of electron proton scattering is given by $\frac{d^{2} \sigma^{e p}}{d Q^{2} d x}=\frac{2 \pi \alpha^{2}}{x Q^{4}}\left[\left(1+(1-y)^{2}\right) F_{2}\left(Q^{2}, x\right)\right]$ for not to large $y$, where $x$ is the fraction of proton momentum carried by the struck quark and $y$ is the inelasticity of the scattering; the structure function $F_{2}$ parameterise the structure of the proton. The structure function $F_{2}^{c \bar{c}}$ and $F_{2}^{b \bar{b}}$ are the contribution to the inclusive structure function $F_{2}$ by events in which a $c \bar{c}$ or $b \bar{b}$ pair has been produced.

Another method to determine these proton structure function $F_{2}^{c \bar{c}}$ and $F_{2}^{b \bar{b}}$ is the so called impact parameter method, were the long lifetime of the heavy meson is used to determine the amount of events with charm or beauty quarks. For the measurement by H1 [7] this methods yields a large visible phase space $\left(1\right.$ track with $\left.p_{t}>500 \mathrm{MeV}\right)$ and a high statistic sample of about 2 Million events. The direction of the quark is estimated either by a jet or rarely by the angle opposite to the scattered electron. Now a positive (negative) sign is applied to the impact parameter $\delta$ of the tracks according whether it crosses the quark flight path after (behind) the primary interaction vertex. Instead of using this signed impact parameter the significance of the track $S=\frac{\delta}{\sigma(\delta)}$ is obtained, where $\sigma(\delta)$ is the error of the impact parameter. Due to the long lifetime of the heavy mesons the significance distribution is asymmetric with a tail to large positive significances, while for light flavors this distribution is nearly symmetric. The negative side is subtracted from the positive side for the distributions $S 1, S 2$ and $S 3$, which contain only the track with highest, second and third highest significance of the event as shown for $S 3$ in Figure 4 . The points are the measurement while the dashed histograms/crosses are monte carlo prediction for charm, beauty and uds production respectively. The prediction are used as templates for a fit in order to describe the shape of the measurement in $S 1, S 2$ and $S 3$.

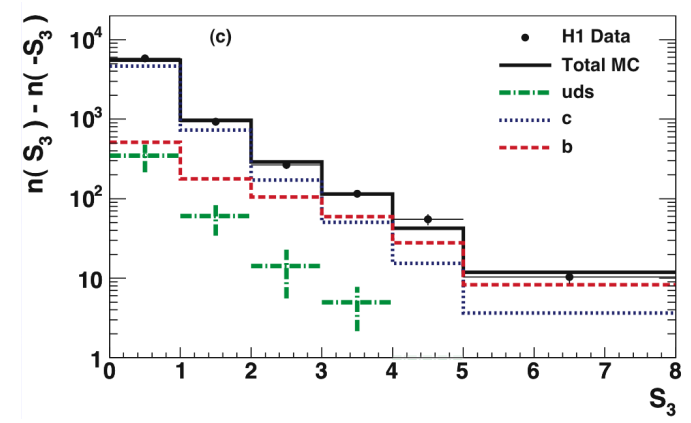

Figure 4: The negative subtracted significance distribution $S 3$ compared to the Monte Carlo predictions.

The so measured cross sections for charm and beauty production can be translated into the proton structure function $F_{2}^{c \bar{c} / b \bar{b}}$, which is shown in the Figures 5 and 6 respectively. These measurements by $\mathrm{H} 1$ are in good agreement compared to measurements by ZEUS which are based upon $D^{\star}, D^{ \pm}$and $p_{t}^{\text {rel }}$ measurements. For the case of $F_{2}^{c \bar{c}}$ the data has reached a high precision and is able to distinguish between the different predic- 
tions especially at low $x$. In the $F_{2}^{b \bar{b}}$ case the statistical errors still dominate, but more data from the HERA II run will be analysed thus decreasing the statistical error significantly. The shown parametrisations for $F_{2}^{b \bar{b}}$ differ up to a factor of two indicating the need of better understanding especially how to match the massive and the massless calculation as it is done for the calculation implementing a VFN scheme.

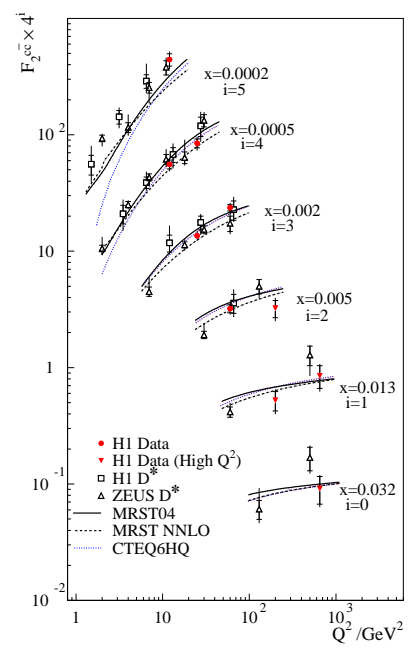

Figure 5: The H1 and ZEUS measurement of $F_{2}^{c \bar{c}}[7]$ as function of the photon virtuality $Q^{2}$ for different Bjorken $x$.

\section{Summary}

A good agreement between data and theory of the charm production in DIS has been achieved. Nevertheless recent measurements reveal deviations in some regions of the phase space which might indicate the need for further theoretical developments. The beauty production is reasonable predicted in shape but the data tend to have higher cross sections than predicted. The extraction of $F_{2}^{c \bar{c} / b \bar{b}}$ by $\mathrm{H} 1$ and ZEUS are in agreement with each other. For charm the error has reached a level that one starts to distinguish

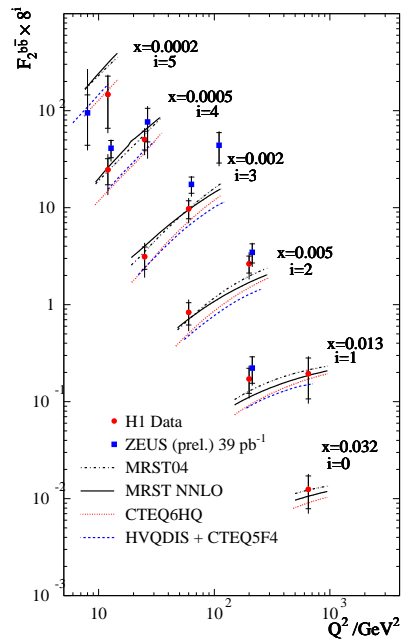

Figure 6: The H1 and ZEUS measurement of $F_{2}^{b \bar{b}}[6]$ as function of the photon virtuality $Q^{2}$ for different Bjorken $x$.

between the different predictions while for beauty the statistical error is still large.

\section{References}

[1] M.-O. Boenig. Slides: http://indico.cern.ch/materialdisplay.py? contribid=56\&amp; sessionid=19 \&amp; materialid=slides\&amp; $\operatorname{confid}=3841$, 2007.

[2] ZEUS Collaboration. F2cc from $D^{ \pm}$Mesons in DIS using 2005 e-p Data. ZEUS-prel-07-008.

[3] H1 Collaboration. $D^{\star}$ Meson Production in Deep Inelastic Scattering at HERA. In EPS, 2007. H1prelim-07-072.

[4] H1 Collaboration. Determination of the Charm Fragmentation Function in Deep-Inelastic Scattering at HERA. In XXII International Symposium on Lepton-Photon Interactions at High Energy, 2005. H1prelim-05-074.

[5] ZEUS Collaboration. Charm Fragmentation Function at HERA. ZEUS-prel-07-010.

[6] ZEUS Collaboration. $F_{2}^{b b}$ at HERAII. ZEUSprel-07-007.

[7] A. Aktas et al. Measurement of F2(c anti-c) and $\mathrm{F} 2$ (b anti-b) at low $\mathrm{Q}^{* *} 2$ and $\mathrm{x}$ using the $\mathrm{H} 1$ vertex detector at HERA. Eur. Phys. J., C45:2333, 2006. 